\title{
Noncatalytic chemical vapor deposition of graphene on high-temperature substrates for transparent electrodes
}

\author{
Jie Sun, ${ }^{1, \text { a) }}$ Matthew T. Cole, ${ }^{2}$ Niclas Lindvall, ${ }^{1}$ Kenneth B. K. Teo, ${ }^{3}$ and August Yurgens ${ }^{1}$ \\ 1) Department of Microtechnology and Nanoscience, Quantum Device Physics Laboratory, \\ Chalmers University of Technology, S-41296 Gothenburg, Sweden \\ ${ }^{2)}$ Department of Engineering, Electrical Engineering Division, University of Cambridge, 9 JJ Thomson Avenue, \\ CB3 OFA Cambridge, United Kingdom \\ 3) AIXTRON Nanoinstruments Ltd., Swavesey, CB24 4 FQ Cambridge, United Kingdom
}

(Dated: 18 December 2011)

A noncatalytic chemical vapor deposition mechanism is proposed, where high precursor concentration, long deposition time, high temperature, and flat substrate are needed to grow large-area nanocrystalline graphene using hydrocarbon pyrolysis. The graphene is scalable, uniform, and with controlled thickness. It can be deposited on virtually any nonmetallic substrate that withstands $\sim 1000{ }^{\circ} \mathrm{C}$. For typical examples, graphene grown directly on quartz and sapphire shows transmittance and conductivity similar to exfoliated or metalcatalyzed graphene, as evidenced by transmission spectroscopy and transport measurements. Raman spectroscopy confirms the $s p^{2}-\mathrm{C}$ structure. The model and results demonstrate a promising transfer-free technique for transparent electrode production.

Graphene, a monolayer of $s p^{2}$ carbon atoms, has received much attention. The use of graphene in transparent electronics is one of its most promising applications. ${ }^{1}$ Apart from its high transparency/conductivity, the flexibility and ease of integration on a variety of substrates are obvious advantages. 30-inch graphene has been demonstrated by virtue of the recent advances in chemical vapor deposition (CVD), ${ }^{2}$ which is compatible with the existing semiconductor technology. ${ }^{3}$ Nevertheless, the necessity of etching the metal catalyst and transfer of graphene to foreign substrates hampers wide industrialization. Thus, efforts are put into the metal-free growth of graphene on materials including $\mathrm{SiO}_{2},{ }^{4-6} \mathrm{Al}_{2} \mathrm{O}_{3},{ }^{7} \mathrm{MgO},{ }^{8}$ etc. To date, however, the graphitization process on dielectrics is poorly understood while the experiments are largely experience based. Recently, we have shown that large-area uniform graphene can be grown on virtually any hightemperature substrates, on $\mathrm{Si}_{3} \mathrm{~N}_{4}$ or $\mathrm{HfO}_{2}$, for instance. ${ }^{9}$ In this letter, we provide a broader and deeper insight, discussing in detail the growth mechanism of graphene directly on insulators. As an example, we show our results on graphene on quartz and sapphire, hinting at the future prospects of the transfer-free graphene for transparent electrodes.

Bulk graphite is usually made at $>3000{ }^{\circ} \mathrm{C}$ and catalysis is needed to lower the temperature. ${ }^{10}$ However, nanoscale graphene flakes form without any catalysts at merely $\sim 1000{ }^{\circ} \mathrm{C}$. For example, carbon black which is produced massively by natural gas pyrolysis ${ }^{11-14}$ is nothing else but chaotically connected graphene flakes. ${ }^{11} \mathrm{Al}-$ though widely used in industry, this process has so far been overlooked for making large-area graphene. Here, we show that macroscopically amorphous carbon black can be turned into textured graphene thin films when

\footnotetext{
a)Electronic mail: jiesu@chalmers.se.
}

the carbon precursor concentration and growth temperature are high, also requiring a relatively long deposition time and flat substrate.

Fig. 1(a) illustrates the CVD reactor used in this work. The middle region is heated to $1000{ }^{\circ} \mathrm{C}$ while the left and right sides are $<600{ }^{\circ} \mathrm{C}$. There is no material deposition in the right zone because of the reduced $\mathrm{CH}_{4}$ decomposition efficiency at lower temperature. Carbon black is seen on the left, as an evidence of the $\mathrm{CH}_{4}$ dissociation in the middle zone. The easiest way to distinguish graphene from carbon black is to observe their optical appearances. The as-grown graphene is smooth and, on any high-temperature substrate, keeps its metallic luster (see Fig. 1(b)) for hundreds of layers, ${ }^{9}$ whereas carbon

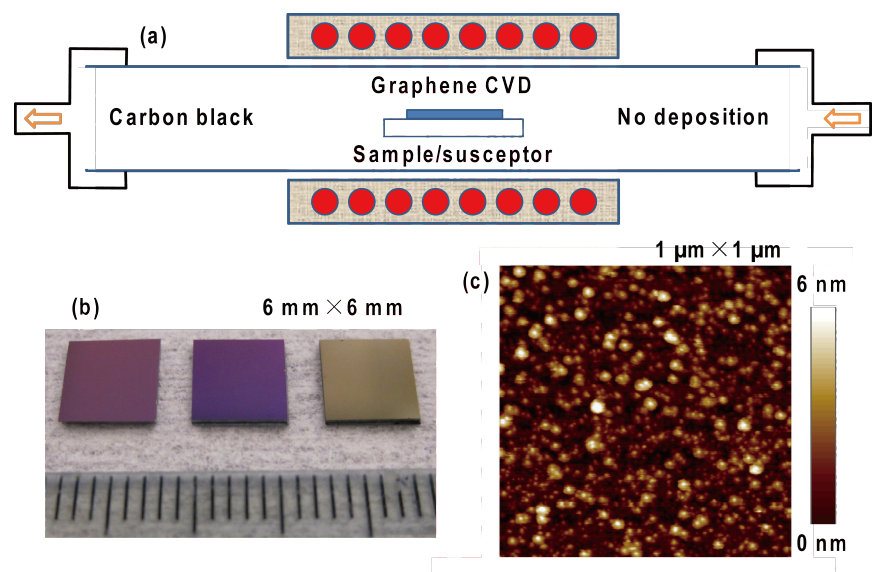

FIG. 1. (Color online) (a) Schematic of the hot-wall CVD system used in this study, indicating zones where graphene and carbon black are deposited. The arrows indicate the direction of gas flow. (b) Photo of bare $300 \mathrm{~nm} \mathrm{SiO}_{2} / \mathrm{Si}$ (left) and with coated nominal monolayer graphene (middle) and $\sim 70$ nm graphite (right). (c) Atomic force microscopy micrograph of the middle sample in (b), where $r_{a} \approx 1 \mathrm{~nm}$. 
black is dull black even for thinner films. Fig. 1(b) shows the photo of (right to left) $\sim 70 \mathrm{~nm}$ thick graphite, monolayer graphene, and the bare substrate $(300 \mathrm{~nm} \mathrm{SiO} 2 / \mathrm{Si})$, respectively. The growth recipe is similar to our previous work, where the thickness is controllable from nominally submonolayer to thick graphite. ${ }^{9}$ Transmission electron microscopy reveals that the middle sample in Fig. 1 (b) is composed of primarily $\sim 10 \mathrm{~nm}$ large monolayer graphene crystallites. ${ }^{15}$ The high density of grain boundaries is fundamental which results in strong scattering of charge carriers and makes such graphene unsuitable for transistors. Nonetheless, this does not reduce its value for applications where transparency of electrodes (e.g. solar cells/displays) or their thickness (e.g. molecular electronics) are more important than the mobility. Indeed, the optical similarity between this graphene and exfoliated- or $\mathrm{Cu}$-catalyzed monolayer graphene ${ }^{16}$ is evidenced by ellipsometry measurements. ${ }^{15}$ Fig. 1(c) is an atomic force microscopy image ${ }^{15}$ of the middle sample in Fig. 1(b). The arithmetic average roughness $r_{a}$ is $\sim 1 \mathrm{~nm}$, which is reasonable for nanocrystalline graphene resting on thermally grown $\mathrm{SiO}_{2}\left(r_{a}\right.$ is $\left.\sim 0.2 \mathrm{~nm}\right)$.

Fig. 2 shows the transmission spectra of nominal monolayer graphene deposited on quartz and sapphire. ${ }^{15}$. The transmittance of graphene is generally independent of the light wavelength, ${ }^{17}$ except a dip at $\sim 270 \mathrm{~nm}$, which is due to the exciton-shifted van Hove singularity in the graphene density of states. ${ }^{18}$ After the coating, the transmittances of quartz and sapphire are reduced by $\sim 2-3 \%$, values similar to exfoliated graphene. ${ }^{17}$ The inset of Fig. 2(a) shows the Fourier transform infrared spectroscopy (FTIR) measurements on the same samples. The spectra have no C-related peaks after CVD, implying atomic-scale thickness. The curves before and after graphene growth are very similar (no additional features in $3000-3700 \mathrm{~cm}^{-1}$ range),${ }^{19,20}$ suggesting the lack of $-\mathrm{OH}$ groups. This offers an advantage over the wet-transferred graphene, where $\mathrm{H}_{2} \mathrm{O}$ is likely to be trapped at the interfaces. The Raman spectra of the graphene on quartz and sapphire are displayed in the inset of Fig. 2(b). The well-defined Raman peaks differentiate our thin films from amorphous $\mathrm{C}(a-\mathrm{C}) .{ }^{21}$ In fact, atomically thin $a$-C films have never been realized by CVD. ${ }^{22}$ The $\mathrm{G}$ band centered at $1600 \mathrm{~cm}^{-1}$ and the 2D band at $2695 \mathrm{~cm}^{-1}$ are well resolved, which are signatures of $s p^{2}$ graphitic materials. D peaks at 1353 $\mathrm{cm}^{-1}$ are also seen. This Raman mode is forbidden in perfect graphitic structure and only becomes active in the presence of disorder. ${ }^{23}$ In our case, the laser spot is $\sim 10 \mu \mathrm{m}$ and covers numerous grain boundaries of the nanocrystalline graphene, which naturally results in large D signals. Consequently, high-order $\mathrm{G}+\mathrm{D}$ bands at 2946 $\mathrm{cm}^{-1}$ are visible. The ratio of D- and G peak heights ${ }^{24}$ allows estimation of the average distance between defects $\sim 7-9 \mathrm{~nm}$, consistent with the grain size $\sim 10 \mathrm{~nm}$ of our thin films. ${ }^{15}$ Hall-bar devices are fabricated from these samples by photolithography. ${ }^{15}$ Room-temperature four-terminal electrical measurements indicate the sheet resistance $R_{s}$ of 2.9 and $13 \mathrm{k} \Omega / \square$ for the quartz- and sapphire-supported graphene, respectively. Again, $R_{s}$ is comparable to that of standard graphene. ${ }^{18}$

Thermal dissociation of methane is described as $\mathrm{CH}_{4}=\mathrm{C}+2 \mathrm{H}_{2}\left(\Delta H^{\circ}=75.6 \mathrm{~kJ} / \mathrm{mol}\right)$. Although $\mathrm{CH}_{4}$ is one of the most stable organic molecules due to a strong $\mathrm{C}-\mathrm{H}$ bond of $440 \mathrm{~kJ} / \mathrm{mol},{ }^{25}$ the reaction readily occurs at $\geq 300$ ${ }^{\circ} \mathrm{C}$ and becomes nearly complete at $\geq 1300{ }^{\circ} \mathrm{C} .{ }^{14}$ When a metal catalyst is present, ${ }^{2,3,16}$ both the hydrocarbon decomposition and graphene formation are boosted. Consequently, graphene grows rapidly at a very low hydrocarbon partial pressure. ${ }^{16}$ Without using metals, which we refer to as "noncatalytic graphene CVD" ${ }^{10,26}$ the deposition requires a much higher methane concentration ( $\sim 200$ vs. $\sim 0.01 \mathrm{mbar}$ ) and longer growth time (30 vs. $5 \min ) \cdot{ }^{9,16}$

The graphene thin-film formation on nonmetallic substrates appears to be different from the mechanism common to e.g. metallic thin films. Indeed, graphene flakes are readily formed in the gas mixture surrounding a hot substrate. The flakes landing on the substrate can have various fates depending on their sizes. Large enough flakes would stick and stay at the surface, reminiscent of the critical-size nuclei in the common thin-film nucleation theory. ${ }^{27}$ The smaller flakes would not adhere

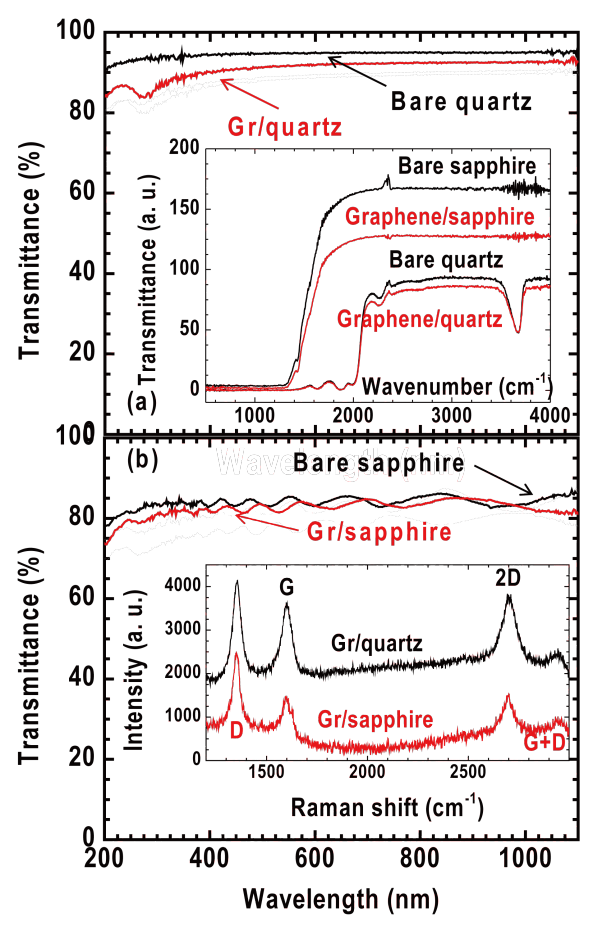

FIG. 2. (Color online) Transmittance spectra of the noncatalytic CVD graphene on (a) quartz and (b) sapphire. The curves in (b) have superimposed interference fringes from the sapphire substrates. Inserts: (a) FTIR spectra of the quartz and sapphire before and after the noncatalytic graphene growth; (b) Raman spectra $(514 \mathrm{~nm}, 3 \mathrm{~mW})$ of the samples. "Gr" denotes graphene. 
to the substrate strongly enough to sustain high-energy thermal vibrations and would leave the substrate, unless they happened to be near the stable flakes ("nuclei") that are already present at the surface and make bonds to them. Stacking of the flakes on top of each other is improbable because the out-of-plane bonding energy between the flakes is lower than their adhesion to the substrate, as in the case of $\mathrm{SiO}_{2},{ }^{28,29}$ impeding formation of the second layer. Reorientation and alignment of the $\mathrm{nm}$-sized flakes requires temperatures $>2000{ }^{\circ} \mathrm{C},{ }^{30}$ and is unlikely to occur in our case, resulting in disordered thin films.

In this picture, the usual growth mechanism, when single atoms have some time to diffuse along the surface and adjoin the stable nuclei, is somehow suppressed, much likely because of the high temperature and the weak adhesion of single atoms to substrate. Next-layers growth, although initially broken short, is still possible after the first graphene layer is formed. Due to a lower grapheneto-graphene cohesion energy (compared with the adhesion to $\left.\mathrm{SiO}_{2}\right),{ }^{28,29}$ larger flakes would now be needed to make stable islands and initiate the growth of the second layer. In principle, the formation of the second- and subsequent layers should then take longer time compared to the first one. However, this difficulty might be compensated for by many defects and dangling bonds at the surface from a nonideality of the first-layer coverage, ${ }^{30}$ resulting in more sites for graphene flake adsorption.

Importantly, all this means that the graphene thin film will grow much quicker with nanoflakes forming already in the gas first, making high precursor pressure and relatively long deposition time crucial. A hot and flat substrate helps preventing large porous lumps of carbon black to form, ${ }^{11-14}$ as there are no sites or bonding strength to attach misplaced flakes with "wrong" orientation. ${ }^{15} \mathrm{~A}$ similar example can be found in liquid phase deposition, where flat substrates help sol particles to form thin films as opposed to the otherwise favorable clumpy precipitates. ${ }^{19,20}$ The discussion above can be generalized to explain graphene growth on any nonmetallic substrate that withstands $\sim 1000{ }^{\circ} \mathrm{C}$ by $\mathrm{CVD}$ using hydrocarbons, not limited to $\mathrm{CH}_{4}$. Obviously, the substrate materials and their preparation play an important role in the growth kinetics. On specially annealed $\mathrm{SiO}_{2}$ where there are possibly less dangling bonds, the growth takes much longer time, but also leads to higher quality of the graphene. ${ }^{31}$

In summary, we have proposed a noncatalytic graphitization mechanism in graphene CVD directly on arbitrary high-temperature insulating substrates. Four experimental conditions-high carbon precursor pressure, long growth time, high temperature, and flat substrate-are needed to modify carbon black deposition into selfassembled nanocrystalline graphene thin film. The asproduced graphene is uniform, scalable, and has properties similar to standard graphene, as confirmed by transmission, FTIR and Raman spectra, as well as transport measurements. This work should stimulate further studies on graphene grown in metal-free processes which are important for applications involving transparent electrodes.

${ }^{1}$ Y. Zhu, Z. Sun, Z. Yan, Z. Jin, and J. M. Tour, ACS Nano 5, $6472(2011)$.

${ }^{2}$ S. Bae, H. Kim, Y. Lee, X. Xu, J.-S. Park, Y. Zheng, J. Balakrishnan, T. Lei, H. R. Kim, Y. I. Song, Y.-J. Kim, K. S. Kim, B. Ozyilmaz, J.-H. Ahn, B. H. Hong, and S. Iijima, Nature Nanotechnol. 5, 574 (2010).

${ }^{3}$ X. Li, W. Cai, J. An, S. Kim, J. Nah, D. Yang, R. Piner, A. Velamakanni, I. Jung, E. Tutuc, S. K. Banerjee, L. Colombo, and R. S. Ruoff, Science 324, 1312 (2009).

${ }^{4}$ T. Takami, S. Ogawa, H. Sumi, T. Kaga, A. Saikubo, E. Ikenaga, M. Sato, M. Nihei, and Y. Takakuwa, E-J. Surf. Sci. Nanotechnol. 7, 882 (2009).

${ }^{5}$ A. Ismach, C. Druzgalski, S. Penwell, M. Zheng, A. Javey, J. Bokor, and Y. Zhang, Nano Lett. 10, 1542 (2010).

${ }^{6}$ J. Hofrichter, B. N. Szafranek, M. Otto, T. J. Echtermeyer, M. Baus, A. Majerus, V. Geringer, M. Ramsteiner, and H. Kurz, Nano Lett. 10, 36 (2010).

${ }^{7}$ Y. Miyasaka, A. Nakamura, and J. Temmyo, Jpn. J. Appl. Phys. 50, $04 \mathrm{DH} 12$ (2011).

${ }^{8}$ M. H. Rummeli, A. Bachmatiuk, A. Scott, F. Borrnert, J. H. Warner, V. Hoffman, J.-H. Lin, G. Cuniberti, and B. Buchner, ACS Nano 4, 4206 (2010).

${ }^{9}$ J. Sun, N. Lindvall, M. T. Cole, K. B. K. Teo, and A. Yurgens, Appl. Phys. Lett. 98, 252107 (2011).

${ }^{10}$ A. Oya and H. Marsh, J. Mat. Sci. 17, 309 (1982).

${ }^{11}$ J. Biscoe and B. E. Warren, J. Appl. Phys. 13, 364 (1942).

${ }^{12}$ L. Fulcheri and Y. Schwob, Int. J. Hydrogen Energy 20, 197 (1995).

${ }^{13}$ N. Z. Muradov and T. N. Veziroglu, Int. J. Hydrogen Energy 30, 225 (2005).

${ }^{14}$ A. W. Weimer, J. Dahl, J. Tamburini, A. Lewandowski, R. Pitts, C. Bingham, and G. C. Glatzmaier, Proceedings of the 2001 US DOE hydrogen program review NREL/CP-570-30535 (2001).

${ }^{15}$ See supplementary material at http://dx.doi.org/10.1063/1.3602921 for details.

${ }^{16}$ J. Sun, N. Lindvall, M. T. Cole, K. T. T. Angel, T. Wang, K. B. K. Teo, D. H. C. Chua, J. Liu, and A. Yurgens, IEEE T. Nanotechnol. in press, doi: 10.1109/TNANO.2011.2160729.

${ }^{17}$ R. R. Nair, P. Blake, A. N. Grigorenko, K. S. Novoselov, T. J. Booth, T. Stauber, N. M. R. Peres, and A. K. Geim, Science 320, 1308 (2008).

${ }^{18}$ F. Bonaccorso, Z. Sun, T. Hasan, and A. C. Ferrari, Nature Photon. 4, 611 (2010).

${ }^{19}$ J. Sun and Y. C. Sun, Chin. J. Chem. 22, 661 (2004).

${ }^{20}$ S. Jie, H. Lizhong, W. Zhaoyang, and D. Guotong, Mat. Sci. Forum 475-479, 1725 (2005).

${ }^{21}$ M. A. Tamor and W. C. Vassell, J. Appl. Phys. 76, 3823 (1994).

22 J. Kotakoski, A. V. Krasheninnikov, U. Kaiser, and J. C. Meyer, Phys. Rev. Lett. 106, 105505 (2011).

${ }^{23}$ A. C. Ferrari and J. Robertson, Phys. Rev. B 61, 14095 (2000).

${ }^{24}$ M. M. Lucchese, F. Stavale, E. H. M. Ferreira, C. Vilani, M. V. O. Moutinho, R. B. Capaz, C. A. Achete, and A. Jorio, Carbon 48, 1592 (2010).

${ }^{25}$ G. I. Kozlov and V. G. Knorre, Combust. Flame 6, 253 (1962).

${ }^{26}$ J. K. Yu, S. Ueno, H. X. Li, and K. Hiragushi, J. Europ. Ceramic Soc. 19, 2843 (1999). Some high-temperature dielectrics can be catalytic in powder form; nevertheless, flat substrates can hardly dissolve or react with $\mathrm{C}$ and therefore play no or weak catalytic role in graphitization.

${ }^{27}$ M. Ohring, Materials Science of Thin Films: Deposition and Structure (Academic Press, San Diego, CA, 2002).

${ }^{28}$ S. P. Koenig, N. G. Boddeti, M. L. Dunn, and J. S. Bunch, Nature Nanotechnol. 6, 543 (2011).

${ }^{29}$ Z. Liu, J. Z. Liu, Y. Cheng, and Q. Zheng, arXiv:1104.1469v1, unpublished.

${ }^{30}$ J. Abrahamson, Carbon 11, 337 (1973). 
${ }^{31}$ J. Chen, Y. Wen, Y. Guo, B. Wu, L. Huang, Y. Xue, D. Geng, D. $\quad$ Wang, G. Yu, and Y. Liu, J. Am. Chem. Soc. 133, 17548 (2011). 